with those for obsessive-compulsive disorder, post-traumatic stress disorder, schizophrenia and personality disorder appealing most to this reviewer. The theoretical underpinnings of cognitive therapy are therefore very well described in this book and this is perhaps its greatest strength.

The research on components of the models and on efficacy is clearly described and where research is lacking this is openly stated. Unfortunately, except in the fields of the anxiety disorders, depression and schizophrenia, the trials undertaken are inadequate to test these very appealing cognitive models. The chapters are often short on critical appraisal of those trials that have been done. In particular, control psychotherapies such as befriending, supportive counselling and social skills training also have very substantial treatment effects in certain trials which are not well explained by current cognitive models. Effectiveness trials are almost non-existent in the cognitive therapy literature so it is unclear as to how or whether cognitive therapy can be delivered in day-to-day clinical practice. This is not adequately acknowledged in the text.

Jesse Wright in his chapter does well to explore the interface between cognitive and biological models in the area of concordance with medication. Although it is encouraging to read a chapter addressing the area of systemic theory and cognition it seems that there is a blind spot on the interface with psychodynamic models. This book is predominantly an excellent exposition of cognitive theory and models but it also highlights the lack of an evidence base for many diagnostic groups. In an era of patient choice, many patients may well choose this treatment modality providing they can be convinced of effectiveness.

This is a stimulating and readable text. It will be widely read by mental health professionals in training, those on cognitive therapy courses and as a refresher text for cognitive therapy practitioners. The book will also stand as an accolade to the vision and perseverence of Aaron T. Beck in devising, testing and promoting this form of psychotherapy.

Douglas Turkington Liaison Psychiatry, Leazes Wing, Royal Victoria Infirmary, Newcastle upon Tyne NEI 4LP. E-mail: douglas.turkington@ncl.ac.uk

\section{Understanding and Treating Fear of Pain}

Edited by Gordon J. G. Asmundson, Johan W. S. Vlaeyen \& Geert Crombez. Oxford: Oxford University Press. 2004. 390pp. $€ 49.50$ (hb).

ISBN 019852514 |

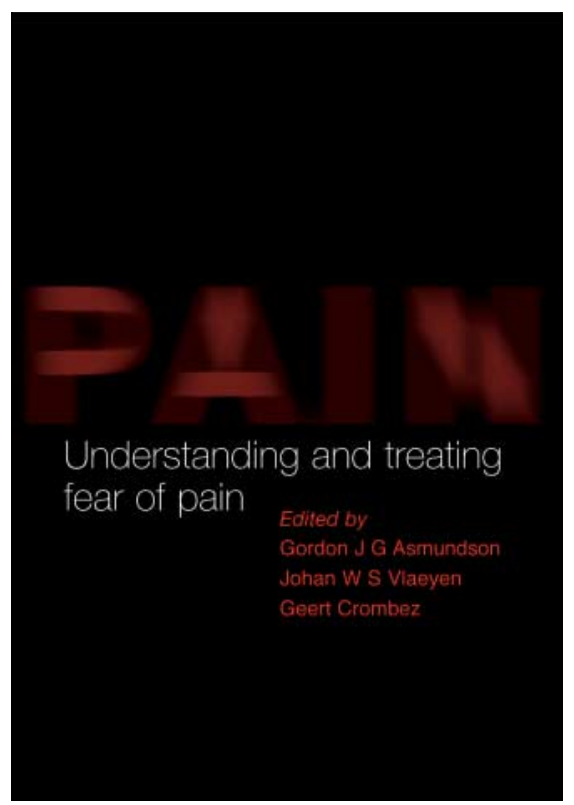

Many of those who develop chronic pain have disproportionately few organic findings to explain their degree of distress. Many of these individuals are classified as suffering from a somatoform disorder. There is debate about the genesis of prolonged pain in these patients.

This book, edited by three psychologists from Canada, Holland and Belgium, posits that fear of pain explains the mechanism of psychological distress in vulnerable patients who become disabled with pain. In those that are predisposed, the perception of pain is interpreted in a malign way through a process of what is described as catastrophisation. The cause of the pain is viewed in a horrific light and there is further rumination and worry about effects on the body. The authors explain that this occurs because of previous life experiences, particularly those of situations involving pain, anxiety and perceived threats to physical integrity. When faced with comparable situations, the sufferer amplifies painful feelings and avoids activities that give rise to further pain. This reduction in mobility limits the process of re-establishment of physical routines that are essential in enabling recovery from injury, and the painful state is reinforced. In others, fear of pain occurs because of fear of anxietyrelated sensations associated with painful episodes, as part of a classical conditioning process.

The authors devote 11 of the 15 chapters in this book to explaining this fear-anxiety-avoidance model of the genesis of chronic pain. This part may appear somewhat convoluted to the scientist grounded in didactic enquiry, but the model described has value in treatment. Specific attention to overcoming fearful cognitions has been found to achieve treatment success in patients with back pain (Von Korff et al, 2005), and these authors contribute one of the four chapters in the treatment section. Graded exposure to anxiety-provoking situations can enable patients to learn to manage both anxiety and pain together. Cognitive-behavioural therapy is also effective.

This book prompts all that are managing patients with painful conditions to examine the nature and quality of the attributions and beliefs of the pain sufferer. Since the book has been published it has been demonstrated that up to $10 \%$ of the disability associated with and intensity of chronic painful complaints can be attributed to fearful cognitions of the sufferers (Peters et al, 2005). It is timely for those involved in treating individuals with chronic pain to consider fear of pain as a possible origin of their distress.

Peters, M. L., Vlaeyen, J.W. S. \& Weber, W. E. J. (2005) The joint contribution of physical pathology, pain-related fear and catastrophizing to chronic back pain disability. Pain, II3, 45-50.

Von Korff, M., Balderson, B. H. K., Saunders, K., et al (2005) A trial of an activating intervention for chronic back pain in primary care and physical therapy settings. Pain, II3, 323-330.

Stephen Tyrer Consultant in Neurorehabilitation and Pain, Department of Psychiatry, Leazes Wing, Royal Victoria Infirmary, Newcastle uponTyne NEI 4LP,UK. E-mail: s.p.tyrer@ncl.ac.uk 